\section{Article}

Doi 10.5943/mycosphere/7/5/11

Copyright (C) Guizhou Academy of Agricultural Sciences

\title{
Checklist of fungi on teak
}

\section{Doilom $\mathrm{M}^{1}$, Taylor $\mathrm{JE}^{2}$, Bhat $\mathrm{DJ}^{3}$, Chukeatirote $\mathbf{E}^{\mathbf{1}}$, Hyde $\mathrm{KD}^{\mathbf{1}}$, To-anun $\mathrm{C}^{4}$ and Jones EBG ${ }^{4,5}$}

${ }^{1}$ Center of Excellence in Fungal Research, and School of Science, Mae Fah Luang University, Chiang Rai 57100, Thailand

${ }^{2}$ Royal Botanic Garden Edinburgh, 20A Inverleith Row, Edinburgh, EH3 5LR, United Kingdom

${ }^{3}$ No. 128/1-J, Azad Housing Society, Curca, Goa Velha, India

${ }^{4}$ Department of Entomology and Plant Pathology, Faculty of Agriculture, Chiang Mai University, Chiangmai 50200, Thailand

${ }^{5}$ Department of Botany and Microbiology, College of Science, King Saud University, P.O. Box: 2455, Riyadh 1145, Saudi Arabia

Doilom M, Taylor JE, Bhat DJ, Chukeatirote E, Hyde KD, To-anun C, Jones EBG 2016 Checklist of fungi on teak. Mycosphere 7(5), 656-678, Doi 10.5943/mycosphere/7/5/11

\begin{abstract}
This publication provides an updated checklist of fungi on teak. This is a compilation of information on substrate and locality from where fungi have been recorded on teak, or original descriptions available. In total, 152 species with 34 hitherto unidentified species are listed here on teak, from 39 countries. The fungi recorded from teak are distributed in 32 orders, 69 families, 134 genera, which can be divided into two taxonomic groups: (i) Ascomycota: 23 orders, 54 families, 114 genera, 132 species identified and 29 unidentified species, and (ii) Basidiomycota: 9 orders, 15 families, 20 genera, 20 species identified and 5 unidentified species.
\end{abstract}

Key words - Ascomycota - Basidiomycota - fungi - Tectona grandis

\section{Introduction}

There are numerous checklists of fungi, either on selected hosts, selected countries, and selected fungal groups. For example, there are checklists of fungi on cabbage trees (Cordyline spp.) and New Zealand flaxes (Phormium spp.) in New Zealand (McKenzie et al. 2005), a checklist of fungi in Panama (Piepenbring 2006), an annotated checklist of smut fungi (Ustilaginomycetes) from Thailand (Shivas et al. 2007), a checklist of aphyllophoraceous fungi in Thailand (Choeyklin et al. 2011), a checklist of rust fungi in Turkey (Bahcecioglu \& Kabaktepe 2012), and a list of fungi associated with Pandanaceae (Whitton et al. 2012). However, there is no comprehensive checklist of fungi associated with Tectona grandis L.f.

In The Systematic Mycology and Microbiology Laboratory (SMML) database (Farr et al. 2016 from https://nt.ars-grin.gov/fungaldatabases/), 151 fungus-host combinations have been recorded on teak. Ascomycota represent most fungi reported from teak and occur as endophytes, pathogens and saprobes (Farr et al. 2016). A few Basidiomycota are also described, and they are mostly associated with rot diseases or basal stem rot on teak (Farr et al. 2016).

Some of fungi on teak are not currently included in the SMML database, such as Daldinia eschscholzii, Fusarium sp., Penicillium sp., Schizophyllum commune and Xylaria sp. (Chareprasert 
et al. 2006). This also includes some lignicolous marine fungi on submerged teak blocks, including Pseudallescheria ellipsoidea (Lu et al. 2000, Vrijmoed et al. 1982, 1986).

Here, a checklist of fungi on teak is presented, and an update of taxa including all fungi recorded during the current study (Doilom et al. 2014, 2015, 2016). This will be a guide for future studies and useful information for the development of a database of fungi on teak.

\section{Materials \& Methods}

The checklist is based on the SMML database (https://nt.ars-grin.gov/fungaldata bases/) (latest accessed 25-9-2016), recent relevant literature and the author's studies. The document includes known hosts and distribution from type or original descriptions available. And information on substrate and locality from where fungi have been recorded on teak, including all those encountered during our own studies (Doilom et al. 2014, 2015, 2016). The current name is used according to Index Fungorum (2016). Genera and species are listed in alphabetical order.

\section{List of fungi associated with Tectona grandis}

\section{Acremonium sp.}

Papua New Guinea and Tanzania, e.g. large concentric leaf blotch on T. grandis (Shaw 1984, Ebbels \& Allen 1979).

Acremonium tectonae R.F. Castañeda [as 'Acromoniun'], Fungi Cubenses II (La Habana): 2 (1987) Cuba, onr leaves of $T$. grandis (type).

Aecidium effusum Niessl, Hedwigia 20: 150 (1881)

India, on leaves of $T$. grandis (type).

Albonectria rigidiuscula (Berk. \& Broome) Rossman \& Samuels, in Rossman, Samuels, Rogerson \& Lowen, Stud. Mycol. 42: 105 (1999)

$\equiv$ Nectria rigidiuscula Berk. \& Broome, J. Linn. Soc., Bot. 14(no. 74): 116 (1873) [1875]

Tanzania, on T. grandis, as Fusarium decemcellulare (Ebbels \& Allen 1979).

Alternaria alternata (Fr.) Keissl., Beih. bot. Zbl., Abt. 2 29: 434 (1912)

$\equiv$ Torula alternata Fr., Syst. mycol. (Lundae) 3(2): 500 (1832)

China, on brown leaf spot of teak (Ai et al. 2015).

\section{Alternaria sp.}

India, Venezuela and Thailand, on leaves of T. grandis (Urtiaga 2004, Chareprasert et al. 2006, Murali et al. 2007).

Alternaria tillandsiae E.G. Simmons \& C.F. Hill, CBS Diversity Ser. (Utrecht) 6: 314 (2007)

USA, on Tillandsia usneoides (type).

Thailand, associated with necrotic leaf lesion of T. grandis (Doilom et al. 2016).

Antennospora quadricornuta (Cribb \& J.W. Cribb) T.W. Johnson, J. Elisha Mitchell scient. Soc. 74: 46 (1958)

$\equiv$ Halosphaeria quadricornuta Cribb \& J.W. Cribb, Pap. Dept. Bot. (formerly Biol.) Univ. Qd. 3: 99 (1956)

Australia, on dead exposed root of Avicennia marina var resinifera (type).

Hong Kong, on wood baits of $T$. grandis (Lu et al. 2000).

Aplosporella beaumontiana S. Ahmad, Biologia, Lahore 8(2): 144 (1962)

Pakistan, on dead branches of Beaumontia grandiflora (type).

India, on T. grandis (Pande \& Rao 1995). 
Aplosporella cesatii Sacc. [as 'cesati'], Syll. fung. (Abellini) 2: 325 (1883)

India, T. grandis (Pande \& Rao 1995).

Armillaria heimii Pegler, Kew Bull., Addit. Ser. 6: 92 (1977)

Madagascar, on dead wood (type).

Zambia, on T. grandis (Gezahgne et al. 2004, Jimu et al. 2015).

Armillaria mellea (Vahl) P. Kumm., Führ. Pilzk. (Zerbst): 134 (1871)

$\equiv$ Agaricus melleus Vahl, Fl. Danic. 6(17): tab. 1013 (1790)

East Indies, Ghana, Indonesia, Malawi, Tanzania and Zambia, on T. grandis (Riley 1960, Spaulding 1961, Anonymous 1964, Corbett 1964, Coetzee et al. 2000, Owusu 2011).

Armillariella mellea (Vahl) P. Karst., Acta Soc. Fauna Flora fenn. 2(no. 1): 4 (1881) [1881-1885]

$\equiv$ Agaricus melleus Vahl, Fl. Danic. 6(17): tab. 1013 (1790)

Malawi, T. grandis (Peregrine \& Siddiqi 1972).

Arthonia radiata (Pers.) Ach., K. Vetensk-Acad. Nya Handl. 29: 131 (1808)

三 Opegrapha radiata Pers., Ann. Bot. (Usteri) 1: 29 (1794)

Cuba, on T. grandis (Urtiaga 1986).

Arthonia sp.

Cuba, on T. grandis (Urtiaga 2004).

Arthrobotrys sp.

Hong Kong, on T. grandis (Lu et al. 2000, Zhuang 2001).

Aschersonia cinnabarina Henn., in Warburg, Monsunia 1: 37 (1899) [1900]

Philippines, on leaves of Glochidion sp. (type).

Myanmar, on leaves of T. grandis (Thaung 2008).

Aspergillus sp.

Hong Kong, on wood baits of T. grandis (Lu et al. 2000, Zhuang 2001).

Asteromella tectonae (Syd., P. Syd. \& E.J. Butler) Aa, in van der Aa \& Vanev, A Revision of the Species Described in Phyllosticta (Utrecht): 452 (2002)

$\equiv$ Phyllosticta tectonae Syd., P. Syd. \& E.J. Butler, Annls mycol. 14(3/4): 181 (1916)

India, on leaves of $T$. grandis, as Phyllosticta tectonae (type).

Myanmar, on leaves of T. grandis, as Asteromella tectonae (Thaung 2008).

Auricularia nigricans (Sw.) Birkebak, Looney \& Sánchez-García, in Looney, Birkebak \& Matheny, N. Amer. Fung. 8(6):12 (2013)

$\equiv$ Exidia polytricha Mont., Voy. Indes Or., Bot. 2: 154 (1834)

Philippines, on T. grandis, as Auricularia polytricha (Teodoro 1937).

Bagnisiella jawaharensis C. Ramesh, Indian Botanical Reporter 5(2): 203 (1987) [1986] India, on bark of $T$. grandis (type).

Barriopsis tectonae Doilom, L.A. Shuttlew. \& K.D. Hyde, in Doilom, Shuttleworth, Roux, Chukeatirote \& Hyde, Phytotaxa 176(1): 84 (2014)

Thailand, on dead branch of $T$. grandis (type).

Berkleasmium talaumae Bat. \& Cavalc., Riv. Patol. veg., Pavia, sér. 3, 4: 565 (1964) 
Brazil, on leaves of Talauma ovata (type).

Thailand, on dead twig of T. grandis (Doilom et al. 2016).

Boerlagiomyces macrospora V.G. Rao \& Varghese [as 'Boerlagomyces'], Sydowia 32(1-6): 254 (1980) [1979]

=Thaxteriella macrospora (V.G. Rao \& Varghese) J.L. Crane, Shearer \& M.E. Barr, Can. J. Bot. 76(4): 606 (1998)

India, on dead wood of dicotyledon plant (type).

Thailand, on dead branches of T. grandis (Doilom et al. 2016).

Bombardia tectonae C. Booth, Mycol. Pap. $94: 7$ (1964)

Jamaica, on calyx of $T$. grandis (type).

Botryobasidium aureum Parmasto, Eesti NSV Tead. Akad. Toim., Biol. seer 14(2): 220 (1965)

= Oidium aureum Link, Mag. Gesell. naturf. Freunde, Berlin 3(1-2): 18 (1809)

Cuba, on T. grandis, as Oidium aureum (Urtiaga 1986).

Brachysporiella rhizoidea (V. Rao \& de Hoog) W.P. Wu, in Wu \& Zhuang, Fungal Diversity Res. Ser. 15: 212 (2005)

三Monotosporella rhizoidea V. Rao \& de Hoog, Stud. Mycol. 28: 6 (1986)

India, on bark of $T$. grandis, as Monotosporella rhizoidea (type).

\section{Capnodium sp.}

Papua New Guinea, on T. grandis (Shaw 1984).

Ceratocladium purpureogriseum B. Sutton, Mysore J. agric. Sci. 7: 401 (1973)

India, on stem of Justicia betonica (type).

Thailand, on dead twig of $T$. grandis (Doilom et al. 2016).

Ceratocystis fimbriata Ellis \& Halst., Bull. New York Agricultural Experimental Station 76: 14 (1890)

Brazil, on stem fragments of T. grandis (Firmino et al. 2012).

Cercospora apii Fresen., Beitr. Mykol. 3: 91 (1863)

China, Hawaii, India, Indonesia, Taiwan, Trinidad and Tobago, on T. grandis (Crous \& Braun 2003).

\section{Cercospora sp.}

Mauritius, on T. grandis (Wiehe 1948).

Cercospora tectonae F. Stevens, Bulletin of the Bernice P. Bishop Museum, Honolulu, Hawaii 19: 155 (1925)

Hawaii, on leaves $T$. grandis (type).

China, Hawaii, India, Indonesia, Taiwan, Thailand, Trinidad and Tobago, e.g. on leaves of T. grandis (Stevens 1925, Chupp 1953, Sawada 1959, Spaulding 1961, Vasudeva 1963, Anonymous 1979, Tai 1979, Baker \& Dale 1951, Dennis 1970, Goos \& Gowing 1992, Chen 2002, Kamal 2010, Meeboon et al. 2007, To-anun et al. 2011).

Cercospora tectoniae F. Stevens, Bulletin of the Bernice P. Bishop Museum, Honolulu, Hawaii 19: 155 (1925)

Hawaii, on leaves $T$. grandis (type). 
Hawaii and Niue, on leaves T. grandis (Anonymous 1960, Dingley et al. 1981, Raabe et al. 1981).

Cercospora tectonigena Kamal \& V.K. Pal, in Kamal, Cercosporoid Fungi of India (Dehra Dun): 90 [+ Errata] (2010)

India, on leaves of $T$. grandis (type).

Chaetomium fusum L.M. Ames, Monograph of the Chaetomiaceae (U.S. Army Research and Development Service): 25 (1963)

= Chaetomium angustisporum C. Booth, Mycol. Pap. 94: 9 (1964).

Jamaica, on calyx of T. grandis, as Chaetomium angustisporum (Booth 1964).

Chaetomium globosum Kunze, in Kunze \& Schmidt, Mykologische Hefte (Leipzig)_1: 16 (1817) Thailand, on dead moist twigs of T. grandis (Maharachchikumbura et al. 2016).

\section{Cladosporium sp.}

China and Hong Kong, on wood baits of T. grandis (Lu et al. 2000, Zhuang 2001).

Cladosporium tectonae Sawada, Rep. Govt Res. Inst. Dep. Agric., Formosa 85: 92 (1943)

Taiwan, on $T$. grandis (type).

Taiwan, on T. grandis (Anonymous 1979, Dugan et al. 2004).

Cladosporium tenuissimum Cooke, Grevillea 6(no. 40): 140 (1878)

South Carolina, on sheaths of Zea Mays (type).

India, on T. grandis (Khan et al. 1989).

Colletotrichum gloeosporioides (Penz.) Penz. \& Sacc., Atti Inst. Veneto Sci. lett., ed Arti, Sér. 6, 2: 670 (1884)

$\equiv$ Vermicularia gloeosporioides Penz., Michelia 2(no. 8): 450 (1882)

India, on leaves of T. grandis, as Colletotrichum gloeosporioides (Murali et al. 2007).

Cuba, on T. grandis, as Glomerella cingulata (Urtiaga 2004).

\section{Colletotrichum sp.}

India, on leaves of T. grandis (Chareprasert et al. 2006, Murali et al. 2007).

Corallomycetella elegans (Berk. \& M.A. Curtis) C. Herrera \& P. Chaverri, Mycosystema 32(3): 533 (2013)

$\equiv$ Corallomyces elegans Berk. \& M.A. Curtis, J. Acad. nat. Sci. Philad., N.S. 2(6): 289 (1854) [1853]

Costa Rica, on canker of T. grandis (Rossman et al. 2013).

\section{Corticium sp.}

Philippines, on T. grandis (Reinking 1920, Teodoro 1937).

Corynespora cassiicola (Berk. \& M.A. Curtis) C.T. Wei, Mycol. Pap. 34: 5 (1950)

$\equiv$ Helminthosporium cassiicola Berk. \& M.A. Curtis [as 'cassiaecola'], in Berkeley, J. Linn. Soc., Bot. 10(no. 46): 361 (1868) [1869]

Cuba, on leaves of Cassia (type).

India, on leaves of $T$. grandis (Murali et al. 2007).

Corynespora tectonae X.G. Zhang \& Ch.K. Shi, Mycotaxon 92: 418 (2005)

China, on dead branches of T. grandis (type). 
Cyanonectria buxi (Fuckel) Schroers, Gräfenhan \& Seifert, in Schroers, Gräfenhan, Nirenberg \& Seifert, Stud. Mycol. 68: 120 (2011)

$\equiv$ Gibbera buxi Fuckel, Jb. nassau. Ver. Naturk. 27-28: 32 (1874) [1873-74]

Germany, branches Buxus sempervirens (type).

India, on T. grandis, as Gibberella buxi (Pande 2008).

\section{Cylindrocephalum sp.}

Hong Kong, on wood baits of T. grandis (Lu et al. 2000, Zhuang 2001).

\section{Cytospora sp.}

Hong Kong, on wood baits of T. grandis (Lu et al. 2000, Zhuang 2001).

Daldinia eschscholzii (Ehrenb.) Rehm [as 'eschscholzii'], Annls mycol. 2(2): 175 (1904)

ESphaeria eschscholtzii Ehrenb., Fung. Champ.: 59, tab. 18, fig. 8 (1820)

Thailand, on leaves of $T$. grandis (Chareprasert et al. 2006).

Diaporthe neoraonikayaporum Doilom, A.J. Dissanayake \& K.D. Hyde, in Doilom et al., Fungal Diversity: 10.1007/s13225-016-0368-7 (2016)

Thailand, associated with branch and twig dieback on $T$. grandis (type).

\section{Diaporthe sp.}

Thailand, on leaves T. grandis (Chareprasert et al. 2006, Udayanga et al. 2012a, b).

Diaporthe tectonae Doilom, A.J. Dissanayake \& K.D. Hyde, in Doilom et al., Fungal Diversity: 10.1007/s13225-016-0368-7 (2016)

Thailand, associated with branch and twig dieback on T. grandis (type).

Diaporthe tectonendophytica Doilom, A.J. Dissanayake \& K.D. Hyde, in Doilom et al., Fungal Diversity: 10.1007/s13225-016-0368-7 (2016)

Thailand, asymptomatic branch of $T$. grandis (type).

Diaporthe tectonigena Doilom, A.J. Dissanayake \& K.D. Hyde, in Doilom et al., Fungal Diversity: 10.1007/s13225-016-0368-7 (2016)

Thailand, associated with twig dieback on $T$. grandis (type).

Diatrype tectonae M.S. Patil \& S.D. Patil, Indian J. Mycol. Plant Path. 13(2): 141 (1985) [1983] India, on bark of $T$. grandis (type).

Diatrypella tectonae M. Doilom, Q.J. Shang \& K.D. Hyde, in Shang et al. (in prep)

Thailand, on dead branch of $T$. grandis (type).

Distoseptispora tectonae Doilom \& K.D. Hyde, in Hyde et al. 2016, Fungal Diversity (in press) Thailand, on dead twig and branch of $T$. grandis (type).

Distoseptispora tectonigena Doilom \& K.D. Hyde, in Hyde et al. 2016, Fungal Diversity (in press) Thailand, on dead twig of $T$. grandis (type).

Dothiorella tectonae Doilom, L.A. Shuttleworth, \& K.D. Hyde, Phytotaxa 233(1): 001-026 (2015) Thailand, on dead branch of $T$. grandis (type).

Ellisembia leonensis (M.B. Ellis) McKenzie, Mycotaxon 56: 13 (1995) 
三Sporidesmium leonense M.B. Ellis, Mycol. Pap. 70: 28 (1958)

Sierra Leone, on dead culms of Pennisetum purpureum (type).

India, on T. grandis, as Sporidesmium leonense (Agarwal et al. 1993).

Erysiphe tectonae (E.S. Salmon) U. Braun \& S. Takam., Schlechtendalia 4: 24 (2000)

$\equiv$ Uncinula tectonae E.S. Salmon, Annls mycol. 5(6): 479 (1907)

India, on $T$. grandis (type).

Myanmar, on leaves of T. grandis, as Erysiphe tectonae (Thaung 2007a).

India and Myanmar, on T. grandis, as Uncinula tectonae (Spaulding 1961, Amano 1986,

Braun 1987, Bappammal et al. 1995, Paul \& Thakur 2006, Pande 2008).

Erythricium salmonicolor (Berk. \& Broome) Burds., Mycol. Mem. 10: 151 (1985)

$\equiv$ Corticium salmonicolor Berk. \& Broome, J. Linn. Soc., Bot. 14(no. 74): 71 (1873) [1875]

Sri Lanka, on bark (type).

India and Indonesia, on T. grandis, as Corticium salmonicolor (Anonymous 1964).

\section{Eutypella sp.}

Papua New Guinea, on dead branch of T. grandis (Shaw 1984).

Fusarium incarnatum (Desm.) Sacc., Syll. fung. (Abellini) 4: 712 (1886)

$\equiv$ Fusisporium incarnatum Desm., Annls Sci. Nat., Bot., sér. 2 10: 309 (1838)

Tanzania, on T. grandis, as Fusarium semitectum (Ebbels \& Allen 1979).

Fusarium solani (Mart.) Sacc., Michelia 2(no. 7): 296 (1881)

$\equiv$ Fusisporium solani Mart., Die Kartoffel-Epidemie der letzten Jahre oder die Stockfäule und Räude der Kartoffeln (Munich) 3: fig. 25-30 (1842)

Tanzania and Thailand, e.g. on stem of T. grandis, as Fusarium solani (Ebbels \& Allen, 1979, Doilom et al. 2016).

India, on T. grandis, as Nectria haematococca (Spaulding 1961).

\section{Fusarium sp.}

Thailand, on leaves of T. grandis (Chareprasert et al. 2006).

Fusicladium tectonicola (Yong H. He \& Z.Y. Zhang) U. Braun \& Bensch, in Schubert, Braun, Groenewald \& Crous, Stud. Mycol. 72(1): 332 (2012)

$\equiv$ Cladosporium tectonicola Yong H. He \& Z.Y. Zhang, Mycosystema 21(1): 21 (2002)

China, on leaves of $T$. grandis, as Cladosporium tectonicola (type).

Cameroon, on leaves of T. grandis, as Fusicladium tectonicola (Braun et al. 2013).

Ganoderma applanatum (Pers.) Pat., Hyménomyc. Eur. (Paris): 143 (1887)

三Boletus applanatus Pers., Observ. mycol. (Lipsiae) 2: 2 (1800) [1799]

India, on T. grandis (Sarbhoy \& Agarwal 1990).

Ganoderma australe (Fr.) Pat., Bull. Soc. mycol. Fr. 5(2,3): 65 (1889)

$\equiv$ Polyporus australis Fr., Elench. fung. (Greifswald) 1: 108 (1828)

Papua New Guinea, on stump of T. grandis, as Ganoderma tornatum (Shaw 1984).

Ganoderma colossus (Fr.) C.F. Baker, Brotéria: 425 (1918)

$\equiv$ Polyporus colossus Fr., Nova Acta R. Soc. Scient. upsal., Ser. 3 1(1): 56 (1851) [1855]

Costa Rica, on stumps of Cedrela odorata (type).

India, on T. grandis (Sarbhoy \& Agarwal 1990). 
Gibberella pulicaris (Kunze) Sacc., Michelia 1(no. 1): 43 (1877)

三Sphaeria pulicaris Kunze, in Kunze \& Schmidt, Mykologische Hefte (Leipzig) 2: 37 (1823)

Tanzania, on T. grandis, as Fusarium sambucinum (Ebbels \& Allen 1979).

Gonatophragmium mori (Sawada) Deighton, in Cejp \& Deighton, Mycol. Pap. 117: 13 (1969)

$\equiv$ Spondylocladium mori Sawada, Spec. Bull. Agric. Exp. Station Formosa 19: 665 (1919)

Taiwan, on Morus alba (type).

Nigeria, on T. grandis (Deighton 1969).

Halosphaeria quadri-remis (Höhnk) Kohlm., Can. J. Bot. 50(9): 1957 (1972)

三 Palomyces quadri-remis Höhnk [as 'quadriremis'], Veröff. Inst. Meeresf. Bremerhaven 3: 213 (1955)

Hong Kong, on wood baits of T. grandis, as Remispora quadri-remis (Lu et al. 2000, Zhuang 2001).

Halosphaeriopsis mediosetigera (Cribb \& J.W. Cribb) T.W. Johnson, J. Elisha Mitchell scient. Soc. 74: 44 (1958)

三 Halosphaeria mediosetigera Cribb \& J.W. Cribb, Pap. Dept. Bot. (formerly Biol.) Univ. Qd. 3: 100 (1956)

Hong Kong, on wood baits of T. grandis, as Trichocladium achrasporum (Lu et al. 2000, Zhuang 2001).

Helicobasidium compactum Boedijn, Arch. voor de Thee Cultuur 1: 10 (1930)

East Indies and Tanzania, on T. grandis (Spaulding 1961, Anonymous 1964, Ebbels \& Allen 1979).

\section{Helicobasidium sp.}

Sudan, on T. grandis (Anonymous 1964).

Helicoma siamense S. Boonmee \& K.D. Hyde, in Boonmee, Rossman, Liu, Crous, Bhat, Chukeatirote, Jones \& Hyde, Fungal Diversity 68: 268 (2014)

Thailand, on dead wood (type).

Thailand, on decaying inner-surface of bark of T. grandis (Doilom et al. 2016).

Hermatomyces tectonae Doilom D.J. Bhat \& K.D. Hyde, in Doilom et al., Fungal Diversity: 10.1007/s13225-016-0368-7 (2016)

Thailand, on dead twigs of $T$. grandis (type).

Hermatomyces thailandica Doilom D.J. Bhat \& K.D. Hyde, in Doilom et al., Fungal Diversity: 10.1007/s13225-016-0368-7 (2016)

Thailand, on dead twigs of $T$. grandis (type).

Huntiella chinaeucensis (S.F. Chen bis, Jol. Roux, M.J. Wingf. \& X.D. Zhou) Z.W. de Beer, T.A. Duong \& M.J. Wingf., in de Beer, Duong, Barnes, Wingfield \& Wingfield, Stud. Mycol. 79: 212 (2014)

三 Ceratocystis chinaeucensis S.F. Chen, M. van Wyk, M.J. Wingf. \& X.D. Zhou, Fungal Diversity 58: 274 (2013)

China, on stumps of E. urophylla $\times$ E. grandis clone (type).

Thailand, on stumps of T. grandis (Maharachchikumbura et al. 2016).

Hyalocladosporiella tectonae Crous \& Alfenas, in Crous et al., Persoonia, Mol. Phyl. Evol. Fungi 32: 237 (2014) 
Brazil, on leaves of $T$. grandis (type).

Hypocrella discoidea (Berk. \& Broome) Sacc., Michelia 1(no. 3): 322 (1878)

$\equiv$ Hypocrea discoidea Berk. \& Broome, J. Linn. Soc., Bot. 14(no. 74): 113 (1873) [1875]

Sri Lanka, on leaves of Zingiber (type).

Myanmar, on Aleyrodes on T. grandis (Thaung 2007b).

Hypoxylon haematostroma Mont., in Sagra, Annls Sci. Nat., Bot., sér. 2, 17: 124 (1842)

Cuba, on fallen bark (type).

India, on dead trunk of T. grandis (Pande 2008).

Kernia ovata (C. Booth) Malloch \& Cain, Mycologia 65(5): 1075 (1973)

$\equiv$ Thielavia ovata C. Booth, Mycol. Pap. 94: 7 (1964)

Jamaica, on calyx of $T$. grandis, as Thielavia ovata (type).

Khuskia oryzae H.J. Huds., Trans. Br. mycol. Soc. 46(3): 358 (1963)

Jamaica, on stems and leaves of Vetiveria zizanioides (type).

Thailand and Venezuela, on leaves of T. gandis, as Nigrospora sphaerica (Urtiaga 2004, Chareprasert et al. 2006).

Kirschsteiniothelia tectonae Doilom, D.J. Bhat \& K.D. Hyde, in Li et al., Fungal Diversity 78:1237 (2016)

Thailand, on dead branches of $T$. grandis (type).

Kretzschmaria deusta (Hoffm.) P.M.D. Martin, J1 S. Afr. Bot. 36(2): 80 (1970)

$\equiv$ Sphaeria deusta Hoffm., Veg. Crypt. 1: 3 (1787)

Tanzania, on T. grandis, as Ustulina deusta (Ebbels \& Allen 1979).

Kretzschmaria zonata (Lév.) P.M.D. Martin, Jl S. Afr. Bot. 42(1): 75 (1976)

ESphaeria zonata Lév., Annls Sci. Nat., Bot., sér. 3 3: 48 (1845)

Nigeria, on T. grandis, as Ustulina zonata (West 1938).

Lasiodiplodia brasiliense M.S.B. Netto et al., in Netto et al., Fungal Diversity 67: 134 (2014)

Brazil, on stems of Mangifera indica (type).

Thailand, on dead branches of $T$. grandis (Doilom et al. 2015, 2016).

Lasiodiplodia pseudotheobromae A.J.L. Phillips et al., Fungal Diversity 28: 8 (2008)

Costa Rica, on Gmelina arborea (type).

Thailand, associated with trunk canker and branch dieback symptoms, and from dead twigs and branches of $T$. grandis (Doilom et al. 2015, 2016).

Lasiodiplodia theobromae (Pat.) Griffon \& Maubl., Bull. trimest. Soc. Mycol. Fr. 25: 57 (1909)

$\equiv$ Botryodiplodia theobromae Pat., Bull. Soc. mycol. Fr. 8(3): 136 (1892)

Tanzania, on T. grandis, as Botryodiplodia theobromae (Ebbels \& Allen 1979).

Brazil, India and Thailand on leaves, dead twig, trunk canker of T. grandis, as Lasiodiplodia theobromae (Murali et al. 2007, Borges et al. 2015).

Latericonis obscura V. Rao, K.A. Reddy \& de Hoog, Mycotaxon 19: 409 (1984)

India, on bark of $T$. grandis (type).

Lectera colletotrichoides (J.E. Chilton) P.F. Cannon, in Cannon, Buddie, Bridge, Neergaard, Lübeck \& Askar, MycoKeys 3: 28 (2012) 
$\equiv$ Volutella colletotrichoides J.E. Chilton, Mycologia 46(6): 801 (1954)

USA, on stem of Medicago sativa (lectotype).

India, on leaf litter of T. grandis (Cannon et al. 2012).

Leptosphaeria compressa (Rehm) L. Holm, Symb. bot. upsal. 14(no. 3): 29 (1957)

$\equiv$ Ophiobolus compressus Rehm, Ber. naturhist. Augsburg 26: 49 (1881)

India, on dead twigs of T. grandis (Pande 2008).

Longiostiolum tectonae Doilom, D.J. Bhat \& K.D. Hyde, in Li et al., Fungal Diversity 78:1-237 (2016)

Thailand, on dead bark of T. grandis (type).

Macrovalsaria megalospora (Mont.) Sivan.Trans. Br. Mycol. Soc. 65: 400 (1975)

三Sphaeria megalospora Mont., Annls Sci. Nat., Bot., sér. 2, 14: 324 (1840)

Several specimens were reexamined in Sivanesan (1975).

Thailand, on dead branches on T. grandis (reference specimen, Doilom et al. 2016).

Manoharachariella tectonae Doilom \& K.D. Hyde, in Doilom et al., Fungal Diversity: 10.1007/s13225-016-0368-7 (2016)

Thailand, on dead branches of $T$. grandis (type).

Melanoctona tectonae Qing Tian, Doilom \& K.D. Hyde, Qing et al. 2016, (In prep) Thailand, on dead branches of $T$. grandis (type).

Moelleriella mollii (Koord.) P. Chaverri, M. Liu \& K.T. Hodge, Stud. Mycol. 60: 3 (2008)

$\equiv$ Hypocrella mollii Koord., Verh. K. Akad. Wet., tweede sect. 13(4): 179 (1907)

Myanmar, on Aleyrodes on T. grandis, as Hypocrella mollii (Thaung 2007b).

Monodictys nitens (Schwein.) S. Hughes, Can. J. Bot. 36: 786 (1958)

三Sporidesmium nitens Schwein., Trans. Am. phil. Soc., New Series 4(2): 306 (1832) [1834]

China, on T. grandis (Zhao \& Zhang 2007, Tianyu 2009).

Monodictys pelagica (T. Johnson) E.B.G. Jones, Trans. Br. mycol. Soc. 46(1): 138 (1963)

$\equiv$ Piricauda pelagica T. Johnson, J. Elisha Mitchell scient. Soc. 74: 42 (1958)

USA, on sunken driftwood (type).

Hong Kong, on wood baits of T. grandis (Lu et al. 2000).

Monodictys sp.

China, on T. grandis (Zhao \& Zhang 2007, Tianyu 2009).

Munkovalsaria donacina (Niessl) Aptroot, Nova Hedwigia 60(3-4): 346 (1995)

= Microthelia donacina Niessl, in Thümen, Contrib. Flor. Mycol. Lusitan.: no. 536 (1879)

Portugal, on dead culms (type).

India, on T. grandis (Aptroot 1995).

Mycosphaerella tecomae F.A. Wolf, Mycologia 35(5): 507 (1943)

Unknow locality, on leaves of Tecoma radicans (type).

Venezuela, on T. grandis (Urtiaga 1986).

Myriangium tectonae Tend., Sydowia 24(1-6): 229 (1971) [1970]

India, on bark of $T$. grandis (type).

Myrothecium roridum Tode, Fung. mecklenb. sel. (Lüneburg) 1: 25 (1790) 
Taiwan, on twigs of $T$. grandis (Matsushima 1980).

Neooccultibambusa chiangraiensis Doilom \& K.D. Hyde, in Doilom et al., Fungal Diversity: 10.1007/s13225-016-0368-7 (2016)

Thailand, on dead twigs $T$. grandis (type).

Nitschkia broomeana (Berk.) Nannf. [as 'broomeiana'], Svensk bot. Tidskr. 69(1): 60 (1975)

$\equiv$ Sphaeria broomeana Berk. [as 'broomeiana'], Hooker's J. Bot. Kew Gard. Misc. 6: 231 (1854)

Sri Lanka, on dead wood (type).

India, on T. grandis (Rajak \& Pandey 1985).

Nitschkia tectonae R.K. Verma, Indian Phytopath. 63(4): 430 (2010)

India, between bark and wood on stem and dead branches of $T$. grandis (type).

\section{Oidiodendron sp.}

Hong Kong, on wood baits of T. grandis (Lu et al. 2000, Zhuang 2001).

\section{Oidium sp.}

Sri Lanka, on T. grandis (Amano 1986).

Olivea tectonae (T.S. Ramakr. \& K. Ramakr.) J.L. Mulder, CMI Descriptions of Pathogenic Fungi and Bacteria 37: no. 365 (1973)

EChaconia tectonae T.S. Ramakr. \& K. Ramakr., Indian Phytopath. 2: 19 (1949)

India, on $T$. grandis (type).

Australia, Brazil, China, Costa Rica, Cuba, India, Indonesia, Pakistan, Panama, Taiwan, Thailand and Viet Nam, e.g. on leaves of T. grandis, as Olivea tectonae (Thirumalachar 1949, Boedijn 1959, Spaulding 1961, Ragunathan \& Ramakrishnan 1972, Anonymous 1979, Tai 1979, Ono \& Hennen 1983, Lorsuwan et al. 1984, Hosagoudar 1988, Zhuang \& Wei 1999, Zhuang 2001, Chen 2002, Arguedas 2004, Daly et al. 2006, Kaneko et al. 2007, Perez et al. 2009, Cabral et al. 2010).

China, India, Indonesia, Japan, Pakistan, Taiwan and Thailand, on T. grandis, as Uredo tectonae (Ito 1950, Spaulding 1961, Giatgong 1980, Chen 2002).

India, Pakistan and Taiwan, on T. grandis, as Chaconia tectonae (Spaulding 1961, Hiratsuka \& Chen 1991).

Ozonium auricomum Link [as 'auriconum'], Mag. Gesell. naturf. Freunde, Berlin 3(1-2): 21 (1809) Philippines, on T. grandis (Teodoro 1937).

Paecilomyces fulvus Stolk \& E.S. Salmon, Persoonia 6(3): 354 (1971)

= Byssochlamys fulva Olliver \& G. Sm., J. Bot., Lond. 71: 196 (1933) 2001).

Hong Kong, on wood baits of T. grandis, as Byssochlamys fulva (Lu et al. 2000, Zhuang

\section{Paecilomyces sp.}

India, on leaves of $T$. grandis (Murali et al. 2007).

Papulaspora halima Anastasiou, Nova Hedwigia 6(3-4): 266 (1963)

USA, on wood of Tamarix aphylla submerged in salt water (type).

Hong Kong, on wood baits of T. grandis (Lu et al. 2000).

Paradictyoarthrinium diffractum Matsush., Matsush. Mycol. Mem. 9: 18 (1996)

South Africa, in stream on a dead decaying spathe of Cocos nucifera (type). 
Thailand, on dead stumps and dead stems of T. grandis (Liu et al. 2015, Doilom et al. 2016).

Paradictyoarthrinium tectonicola Doilom \& K.D. Hyde, in Liu et al., Fungal Diversity. 72: 1-197 (2015)

Thailand, on dead stem of $T$. grandis (type).

Parascedosporium tectonae (C. Booth) Gilgado, Gené, Cano \& Guarro, Int. J. Syst. Evol. Microbiol. 57(9): 2176 (2007)

$\equiv$ Graphium tectonae C. Booth, Mycol. Pap. 94: 5 (1964)

Jamaica, on seeds T. grandis, as Graphium tectonae (type, Lackner \& de Hoog 2011).

Patellaria atrata (Hedw.) Fr., Syst. mycol. (Lundae) 2(1): 158 (1822)

$\equiv$ Lichen atratus Hedw., Descr. micr.-anal. musc. frond. 2(3): 61 (1788) [1789]

India, on dead woods of T. grandis (Pande 2008).

\section{Penicillium sp.}

Thailand, on leaves of T. grandis (Chareprasert et al. 2006).

Periconia prolifica Anastasiou, Nova Hedwigia 6(3-4): 260 (1963)

USA, on wood of Tamarix aphylla submerged in salt water (type).

Hong Kong, on wood baits of T. grandis (Lu et al. 2000).

\section{Pestalotia spp.}

India, on leaves of T. grandis (Mathur 1979).

Pestalotiopsis kwangsiensis Y.X. Chen \& G. Wei, in Chen, Wei \& Chen, Mycosystema 21(3): 319 (2002)

China, on leaves of Sinopimelodendron kuwangsiensis (type).

China, on T. grandis (Ge et al. 2009).

\section{Pestalotiopsis sp.}

India, on leaves of $T$. grandis (Murali et al. 2007).

Petriella setifera (Alf. Schmidt) Curzi, Boll. R. Staz. Patalog. Veget. Roma, N.S. 10: 34 (1930)

三Microascus setifer Alf. Schmidt, Die Verbreitung der coprophilen Pilze in Schlesien: 30 (1912)

Hong Kong, on wood baits of T. grandis (Lu et al. 2000).

Peyronellaea obtusa (Fuckel) Aveskamp, Gruyter \& Verkley, in Aveskamp, Gruyter, Woudenberg, Verkley \& Crous, Stud. Mycol. 65: 33 (2010)

$\equiv$ Phoma obtusa Fuckel, Bot. Ztg. 27:82 (1869)

Tanzania, on T. grandis, as Botryosphaeria obtusa (Ebbels \& Allen 1979).

Phaeoacremonium italicum A. Carlucci \& M.L. Raimondo, in Raimondo, Lops \& Carlucci, Mycologia 106(6): 1123 (2014)

Italy, on Vitis vinifera (type).

Thailand, associated with stem wilt of T. grandis (Doilom et al. 2016).

Phaeoacremonium tectonae Doilom \& K.D. Hyde, Fungal Diversity, Ariyawansa in et al., Fungal Diversity 75: 27-274.

Thailand, associated with twig heart rot and stem wilt of $T$. grandis (type). 
Phellinidium lamaoense (Murrill) Y.C. Dai [as 'lamaënse'] Ann. bot. fenn. 32(1): 69 (1995)

三Pyropolyporus lamaoensis Murrill [as 'lamaensis'], Bull. Torrey bot. Club 34: 479 (1907)

Philippines, on decayed wood (type).

East Indies, on T. grandis, as Fomes lamaoensis (Spaulding 1961, Anonymous 1964).

Phellinus noxius (Corner) G. Cunn., Bull. N.Z. Dept. Sci. Industr. Res., Pl. Dis. Div. 164: 221 (1965)

$\equiv$ Fomes noxius Corner, Gardens' Bulletin, Strait Settlements 5(12): 324 (1932)

Malaysia, on Hevea brasiliensis (type).

Papua New Guinea and Malaysia, on stump and causing basal root rot on T. grandis, as Phellinus noxius (Shaw 1984, Mohd Farid et al. 2005a, b).

Africa, Indonesia, Nigeria, on T. grandis, as Fomes noxius (Anonymous 1964, West 1938.)

Phialophora sp.

Hong Kong, on wood baits of T. grandis (Lu et al. 2000, Zhuang 2001).

\section{Phoma sp.}

Guinea, India and Tanzania, e.g. on leaves of T. grandis (Kranz 1965, Murali et al. 2007, Ebbels \& Allen 1979).

\section{Phomopsis sp.}

India, on leaves of $T$. grandis (Murali et al. 2007).

Phomopsis tectonae D.P. Tiwari, R.C. Rajak \& Nikhra, Curr. Sci. 50(22): 1002 (1981)

India, on leaves of $T$. grandis (type).

Venezuela, on T. grandis (Urtiaga 1986).

Phyllactinia guttata (Wallr.) Lév., Annls Sci. Nat., Bot., sér. 3 15: 144 (1851)

$\equiv$ Alphitomorpha guttata Wallr., Verh. Ges. nat. Freunde Berlin 1(1): 42 (1819) India, on T. grandis (Spaulding 1961).

Phyllosticta capitalensis Henn., Hedwigia 48: 13 (1908)

Brazil, on leaves of Stanhopea sp. (type).

India and Thailand, on leaves of T. grandis (Murali et al. 2007, Wikee et al. 2013a, b).

\section{Phyllosticta sp.}

Cuba, Venezuela, on T. grandis (Urtiaga 1986, 2004).

Podosordaria nigripes (Klotzsch) P.M.D. Martin, Jl S. Afr. Bot. 42(1): 80 (1976)

三Sphaeria nigripes Klotzsch, Linnaea 7: 203 (1832)

India, on ground (type).

India, on T. grandis, as Xylaria nigripes (Pande 2008).

Prosthecium tectonae (Tilak \& R. Rao) A. Pande, Ascomycetes of Peninsular India (Jodhpur): 290 (2008)

$\equiv$ Calospora tectonae Tilak \& R. Rao, (1966)

India, on dead branches of T. grandis, as Calospora tectonae (type).

Pseudallescheria ellipsoidea (Arx \& Fassat.) McGinnis, A.A. Padhye \& Ajello [as 'ellipsoideum'], Mycotaxon 14(1): 98 (1982)

$\equiv$ Petriellidium ellipsoideum Arx \& Fassat., in Arx, Persoonia 7(3): 370 (1973)

Hong Kong, on wood baits of T. grandis (Lu et al. 2000). 
Pseudocercospora pallida (Ellis \& Everh.) H.D. Shin \& U. Braun, Mycotaxon 74(1): 114 (2000)

$\equiv$ Cercospora pallida Ellis \& Everh., J. Mycol. 3(2): 21 (1887)

USA, on living leaves of Tecoma radicans (type).

India, on T. grandis (Kamal 2010).

\section{Pseudocercospora sp.}

Venezuela, on T. grandis (Urtiaga 2004).

Pseudocercospora tectonicola J.M. Yen, A.K. Kar \& B.K. Das, Mycotaxon 16(1): 68 (1982)

India, on leaves of $T$. grandis (type).

India and Laos, on leaves of T. grandis (Yen 1982, Kamal 2010, Phengsintham et al. 2010).

Pseudocoleodictyospora sukhothaiensis Doilom \& K.D. Hyde, in Doilom et al., Fungal Diversity: 10.1007/s13225-016-0368-7 (2016)

Thailand, on bark of living $T$. grandis (type).

Pseudocoleodictyospora tectonae Doilom \& K.D. Hyde, in Doilom et al., Fungal Diversity: 10.1007/s13225-016-0368-7 (2016)

Thailand, on dead bark of $T$. grandis (type).

Pseudocoleodictyospora thailandica Doilom \& K.D. Hyde, in Doilom et al., Fungal Diversity: 10.1007/s13225-016-0368-7 (2016)

Thailand, on bark of living $T$. grandis (type).

Pseudofusicoccum adansoniae Pavlic et al., Mycologia 100(6): 855 (2008)

Western Australia, on dying branch of Adansonia gibbosa (type).

Thailand, associated with leaf spots of T. grandis (Doilom et al. 2015).

Pseudomonodictys tectonae Doilom \& K.D. Hyde, Fungal Diversity, Ariyawansa in et al., Fungal Diversity 75: 27-274.

Thailand, dead wood of T. grandis (type, Doilom et al. 2016).

Ramichloridium apiculatum (J.H. Mill., Giddens \& A.A. Foster) de Hoog, Stud. Mycol. 15: 69 (1977)

三Chloridium apiculatum J.H. Mill., Giddens \& A.A. Foster, Mycologia 49(6): 789 (1958) [1957]

USA, from forest soil (type).

India, on T. grandis (de Hoog 1977).

\section{Rhizoctonia sp.}

Tanzania, on T. grandis (Ebbels \& Allen 1979).

Rhytidhysteron tectonae Doilom \& K.D. Hyde, in Doilom et al., Fungal Diversity: 10.1007/s13225-016-0368-7 (2016)

Thailand, on dead branches of $T$. grandis (type).

Rigidoporus lineatus (Pers.) Ryvarden, Norw. J1 Bot. 19: 236 (1972)

$\equiv$ Polyporus zonalis Berk., Ann. Mag. nat. Hist., Ser. 1 10: 375 (1843) [1842]

Sri Lanka (type).

India, Puerto Rico and Virgin Islands, on T. grandis, as Polyporus zonalis (Anonymous 1964, Stevenson 1975). 
Rigidoporus microporus (Sw.) Overeem, Icon. Fung. Malay. 5: 1 (1924)

$\equiv$ Boletus microporus Sw., Fl. Ind. Occid. 3: 1925 (1806)

Jamaica (type).

Nigeria, on T. grandis, as Fomes lignosus (West 1938).

Rosellinia dimidiata Starbäck, Bih. K. svenska VetenskAkad. Handl., Afd. 3 25(no. 1): 49 (1899)

India, on roots of $T$. grandis (Pande 2008).

Sarcinella tectonae Hosag., Zoos' Print Journal 19(3): 1386-1389 (2004)

India, on $T$. grandis (type).

Schiffnerula tectonae (Thite \& C.R. Patil) Hosag., Zoos' Print Journal 18(4): 1077 (2003)

$\equiv$ Clypeolella tectonae Thite \& C.R. Patil, Geophytology 15(1): 85 (1985)

India, on leaves of $T$. grandis (type, Hosagoudar 2011).

Schizophyllum commune Fr. [as 'Schizophyllus communis'], Observ. mycol. (Havniae) 1: 103 (1815)

Thailand, on leaves of T. grandis (Chareprasert et al. 2006).

Sphaceloma tectonae Wani \& Thirum., Sydowia 23(1-6): 263 (1970) [1969]

India, on leaves and shoots of $T$. grandis (type).

Sphaeropsis eucalypticola A.J.L. Phillips, Persoonia, Mol. Phy,. Evol. Fungi 76:158 (2013)

= Phaeobotryosphaeria eucalypti Doilom et al., Fungal Diversity 57(1): 190 (2012)

Thailand, on dead twig of Eucalyptus (type).

Thailand, on dead branch of $T$. grandis (Doilom et al. 2016).

Sporormiella sp.

India, on leaves of $T$. grandis (Murali et al. 2007).

Sporotrichum sp.

Hong Kong, on wood baits of T. grandis (Lu et al. 2000, Zhuang 2001).

Stachybotrys echinata (Rivolta) G. Sm., Trans. Br. mycol. Soc. 45(3): 392 (1962)

$\equiv$ Penicillium echinatum Rivolta, in Torino \& Speirani, Dei parassiti vegetali .?: 451 (1873)

Taiwan, on leaves of T. grandis, as Memnoniella echinata (Matsushima 1980).

Stachybotrys levispora (Subram.) Yong Wang bis, K.D. Hyde, McKenzie, Y.L. Jiang \& D.W. Li, in Wang, Hyde, McKenzie, Jiang, Li \& Zhao, Fungal Diversity 71: 57 (2015)

$\equiv$ Memnoniella levispora Subram., J. Indian bot. Soc. 33: 40 (1954)

India, on a dead stem (type).

Thailand, on dead branch and dead twigs of T. grandis (Doilom et al. 2016).

Stachybotrys renispora P.C. Misra, Mycotaxon 4(1): 161 (1976)

India, on seeds of Phlox drummondii (type).

Thailand, dead twig of T. grandis (Doilom et al. 2016).

Stemphylium sphaericum Sacc., Atti Accad. Sci. Ven.-Trent.-Istr. 10: 86 (1917)

= Hermatomyces sphaericus (Sacc.) S. Hughes, Mycol. Pap. 50: 100 (1953)

Philippines, on branches Barleria cristata (type).

China, on T. grandis, as Hermatomyces sphaericus (Tianyu 2009). 
Subglobosporium tectonae Doilom \& K.D. Hyde, in Doilom et al., Fungal Diversity: 10.1007/s13225-016-0368-7 (2016)

Thailand, on bark of dead and living teak of T. grandis (type).

Taeniolella muricata (Ellis \& Everh.) S. Hughes, Can. J. Bot. 36: 817 (1958)

$\equiv$ Dendryphion muricatum Ellis \& Everh. [as 'Dendryphium'], Proc. Acad. nat. Sci. Philad. 43: 92 (1891)

USA, on wood of Prunus virginiana (type).

India, on T. grandis (Agarwal et al. 1993).

Talaromyces funiculosus (Thom) Samson, N. Yilmaz, Frisvad \& Seifert, in Samson, Yilmaz, Houbraken, Spierenburg, Seifert, Peterson, Varga \& Frisvad, Stud. Mycol. 70: 176 (2011)

$\equiv$ Penicillium funiculosum Thom, Bull. U.S. Department of Agriculture, Bureau Animal Industry 118: 69 (1910)

Tanzania, on T. grandis, as Penicillium funiculosum (Ebbels \& Allen 1979).

Thanatephorus cucumeris (A.B. Frank) Donk, Reinwardtia 3: 376 (1956)

$\equiv$ Hypochnus cucumeris A.B. Frank, Ber. dt. bot. Ges. 1: 62 (1883)

Papua New Guinea, on seedling and causing leaf blight of T. grandis (Shaw 1984).

Thaxteriellopsis lignicola Sivan., Panwar \& S.J. Kaur, Kavaka 4: 39 (1977) [1976]

$\equiv$ Chaetosphaerulina lignicola (K.S. Panwar \& S.J. Kaur), J.L. Crane, Shearer \& M.E. Barr, Can. J. Bot. 76(4): 608 (1998)

India, on dead wood of Lingo emortuo (type).

Thailand, on decaying inner-surface of bark of T. grandis (Doilom et al. 2016).

Thielaviopsis basicola (Berk. \& Broome) Ferraris, Fl. ital. crypt., Fungi 1(8): 233 (1912)

$\equiv$ Torula basicola Berk. \& Broome, Ann. Mag. nat. Hist., Ser. 2 5: 461 (1850)

Brazil, on T. grandis (Borges et al. 2014).

Trametes elegans (Spreng.) Fr., Epicr. syst. mycol. (Upsaliae): 492 (1838) [1836-1838]

$\equiv$ Daedalea elegans Spreng., K. svenska Vetensk-Akad. Handl. 41: 51 (1820)

Puerto Rico and Virgin Islands, on dead wood of $T$. grandis, as Daedalea elegans (Stevenson 1975).

Trichocladium indicum V.G. Rao, K.A. Reddy, D.R. Kumar \& B.S. Reddy, Indian J. Bot. 8(2): 154 (1985)

India, on bark of $T$. grandis (type).

Trichoderma harzianum Rifai, Mycol. Pap. 116: 38 (1969)

England, from soil (type).

Tanzania, on T. grandis (Ebbels \& Allen 1979).

\section{Trichoderma sp.}

Hong Kong, on wood baits of T. grandis (Lu et al. 2000, Zhuang 2001).

Tubeufia tectonae Doilom \& K.D. Hyde, in Doilom et al., Fungal Diversity: 10.1007/s13225-0160368-7 (2016)

Thailand, on decaying inner-surface of bark of $T$. grandis (type).

\section{Verticillium sp.}

Hong Kong, on wood baits of T. grandis (Lu et al. 2000, Zhuang 2001). 
Wiesneriomyces laurinus (Tassi) P.M. Kirk, Trans. Br. mycol. Soc. 82(4): 748 (1984)

三Volutellaria laurina Tassi, Atti R. Accad. Fisiocrit. Siena, Sér. 4 8: 5 (1897)

Taiwan, on leaves of T. grandis, as Wiesneriomyces javanicus (Matsushima 1980).

Xylaria humosa Lloyd, Mycol. Writ. 7(Letter 68): 1179 (1923)

India, on fallen tree trunks of T. grandis (Pande 2008).

Xylaria longipes Nitschke, Pyrenomyc. Germ. 1: 14 (1867)

United Kingdom, on rotten log of Acer pseudoplatanus (type).

India, on wood of T. grandis (Pande 2008).

Xylaria pallida Berk. \& Cooke, J. Linn. Soc., Bot. 15: 395 (1876) [1877]

Peru, (type).

India, on dead wood of T. grandis (Pande 2008).

Xylaria tectonae A. Pande \& Waing., J. Econ. Taxon. Bot. 28(3): 612 (2004)

India, on logs of $T$. grandis (type).

Xylaria sp.

Thailand, on leaves of T. grandis (Chareprasert et al. 2006).

Xylaria thwaitesii Berk. \& Cooke, Grevillea 12(no. 61): 1 (1883)

Sri Lanka, on wood (type).

East Indies and Indonesia, on T. grandis (Spaulding 1961, Anonymous 1964).

\section{Conclusions}

This is an updated worldwide checklist of fungi on teak. These taxa are distributed in 32 orders, 69 families, 134 genera, 152 species with 34 unidentified species according to the information from the SMML database and relevant literature. The most commonly reported genus on teak is Xylaria with five known species (X. humosa, X. longipes, X. pallida, X. tectonae and X. thwaitesii) and one unidentified taxon. Cercospora is the next common genus with four known species including $C$. apii, $C$. tectonae, $C$. tectoniae, $C$. tectonigena and one unidentified species. Olivea tectonae is reported as the most widely distributed species in 13 countries (Australia, Brazil, China, Costa Rica, Cuba, India, Indonesia, Japan, Pakistan, Panama, Taiwan, Thailand and Viet Nam). The largest number of fungi are reported (57)-from India (57), and then Thailand with 55 taxa. There were 39 species epithets synonymised to current names according to Index Fungorum (2016) including 29 species of Ascomycota and 10 species of Basidiomycota.

\section{Acknowledgements}

This work was financially supported by the Thailand Research Fund through the Royal Golden Jubilee Ph.D. Program grant (No. Ph.D./0072/2553 in 4.S.M.F./53/A.2). Mae Fah Luang University (grant for study Dothideomycetes No. 56101020032) is also thanked for funding laboratory work. We deeply thank the Royal Botanic Garden Edinburgh Library, Edinburgh, United Kingdom for access to useful literatures.

\section{References}

Agarwal GP, Gupta S, Pandey AK. 1993 - Saprophytic fungi from Jabalpur, New additions. Journal of Economic and Taxonomic Botany 17, 79-87. 
Ai C, Wu F, Sun S, Shan T, Wang J. 2015 - First report of brown leaf spot caused by Alternaria alternate on Teak in China. Plant disease 99, p 887.

Amano K. 1986 - Host range and geographical distribution of the powdery mildew fungi. Japan Scientific Societies Press, Tokyo, p 398.

Anonymous. 1960 - Index of Plant Diseases in the United States. USDA Agriculture Handbook no. $165,1-531$.

Anonymous. 1964 - Diseases of Widely Planted Forest Trees. U.S. Department of Agriculture, Forest Service, 237.

Anonymous. 1979 - List of Plant Diseases in Taiwan. Plant Protection Society, Republ of China, $1-404$.

Aptroot A. 1995 - Redisposition of some species excluded from Didymosphaeria (Ascomycotina). Nova Hedwigia 60, 325-379.

Arguedas M. 2004 - La roya de la tecta Olivea tectonae (Rac.): consideraciones sobre su presencia en Panamá y Costa Rica. Kurú: Revista Forestal (Costa Rica) 1, 1-6.

Ariyawansa HA, Hyde KD, Jayasiri SC, Buyck B, Chethana KWT, Dai DQ, Dai YC, Daranagama DA, Jayawardena RS, Lücking R, Ghobad-Nejhad M, Niskanen T, Thambugala KM, Voigt K, Zhao RL, Li GJ, Doilom M, Boonmee S, Yang ZL, Cai Q, Cui YY, Bahkali AH, Chen J, Cui BK, Chen JJ, Dayarathne MC, Dissanayake AJ, Ekanayaka AH, Hashimoto A, Hongsanan S, Jones EBG, Larsson E, Li WJ, Li QR, Liu JK, Luo ZL, Maharachchikumbura SSN, Mapook A, McKenzie EHC, Norphanphoun C, Konta S, Pang KL, Perera RH, Phookamsak R, Phukhamsakda C, Pinruan U, Randrianjohany E, Singtripop C, Tanaka K, Tian CM, Tibpromma S, Abdel-Wahab MA, Wanasinghe DN, Wijayawardene NN, Zhang JF, Zhang H, Abdel-Aziz FA, Wedin M, Westberg M, Ammirati JF, Bulgakov TS, Lima DX, Callaghan TM, Callac P, Chang CH, Coca LF, Dal-Forno M, Dollhofer V, Fliegerová K, Greiner K, Griffith GW, Ho HM, Hofstetter V, Jeewon R, Kang JC, Wen TC, Kirk PM, Kytövuori I, Lawrey JD, Xing J, Li H, Liu ZY, Liu XZ, Liimatainen K, Lumbsch HT, Matsumura M, Moncada B, Nuankaew S, Parnmen S, de Azevedo Santiago ALCM, Sommai S, Song Y, de Souza, CAF, de Souza-Motta CM, Su HY, Suetrong S, Wang Y, Wei SF, Wen TC, Yuan HS, Zhou LW, Réblová M, Fournier J, Camporesi E, Luangsa-ard JJ, Tasanathai K, Khonsanit A, Thanakitpipattana D, Somrithipol S, Diederich P, Millanes AM, Common RS, Stadler M, Yan JY, Li X, Lee HW, Nguyen TTT, Lee HB, Battistin E, Marsico O, Vizzini A, Vila J, Ercole E, Eberhardt U, Simonini G, Wen HA, Chen XH. 2015a - Fungal diversity notes 111-252-taxonomic and phylogenetic contributions to fungal taxa. Fungal Diversity 75, 27-274.

Bahcecioglu Z, Kabaktepe S. 2012 - Checklist of rust fungi in Turkey. Mycotaxon 119, 1-81.

Bappammal M, Hosagoudar VB, Udaiyan K. 1995 - Powdery mildews of Tamil Nadu, India. New Botanist 22, 81-175.

Baker RED, Dale WT. 1951 - Fungi of Trinidad and Tobago. Mycological Papers 33, 1-123.

Boedijn KB. 1959 - The Uredinales of Indonesia. Nova Hedwigia 1, 463-496.

Booth C. 1964 - Studies of Pyrenomycetes: VII. Mycological Papers 94, 1-16.

Borges RCF, Santos MDM, Macedo MA, Martins I, Nascimento AG, Boiteux LS, Fonseca MEN, Mello SCM. 2014 - First report of a wilt disease of Tectona grandis caused by Thielaviopsis basicola in Brazil. New Disease Reports 30, 17.

Borges RCF, Santos MDM, Macedo MA, Martins I, Nascimento AG, Café-Filho AC, Boiteux LS, Fonseca MEN, Inácio CA, Mello SCM. 2015 - A trunk canker disease of Tectona grandis induced by Lasiodiplodia theobromae in Brazil. New Disease Reports 31, 26.

Braun U. 1987 - A monograph of the Erysiphales (powdery mildews). Beih. Nova Hedwigia 89, 1700.

Braun U, Piatek M, Scheuer C. 2013 - New species and new records of several phytopathogenic hyphomycetes. Schlechtendalia 25, 41-48. 
Cabral PGC, Capucho AS, Pereira OL, Maciel-Zambolim E, Freitas RL, Zambolim L. 2010 - First report of teak leaf rust disease caused by Olivea tectonae in Brazil. Australasian Plant Disease Notes 5, 113-114.

Cannon PF, Buddie AG, Bridge PD, de Neergaard E, Lubeck M, Askar MM. 2012 - Lectera, a new genus of the Plectosphaerellaceae for the legume pathogen Volutella colletotrichoides. MycoKeys 3, 23-36.

Chareprasert S., Piapukiew J, Thienhirun S, Whalley AJS, Sihanonth P. 2006 - Endophytic fungi of teak leaves Tectona grandis L. and rain tree leaves Samanea saman Merr. World Journal of Microbiology and Biotechnology 22, 481-486.

Chen MM. 2002 - Forest fungi phytogeography, Forest fungi phytogeography of China, North America, and Siberia and international quarantine of tree pathogens. Pacific Mushroom Research and Education Center, Sacramento, California,USA.

Choeyklin R, Hattori T, Jones EBG. 2011 - A checklist of aphyllophoraceous fungi in Thailand, Part I. New records. Mycosphere 2, 161-177.

Chupp C. 1953 - Monograph of the fungus genus Cercospora. Published by the Author, Ithaca, New York, 1-667.

Coetzee MPA, Wingfield BD, Coutinho TA, Wingfield MJ. 2000 - Identification of the causal agent of Armillaria root rot of Pinus species in South Africa. Mycologia 92, 777-785.

Corbett DCM. 1964 - A supplementary list of plant diseases in Nyasaland. Mycological Papers 95, $1-16$.

Crous PW, Braun U. 2003 - Mycosphaerella and its anamorphs, 1. Names published in Cercospora and Passalora. Centraalbureau voor Schimmelcultures, Utrecht, 571.

Daly AM, Shivas RG, Pegg GS, Mackie AE. 2006 - First record of teak leaf rust (Olivea tectonae) in Australia. Australasian Plant Disease Notes 1, 25-26.

de Hoog GS. 1977 - Rhinocladiella and allied genera. Studies in Mycology 15, 1-140.

Deighton FC. 1969 - New genera and species and redispositions of some hyphomycetes, mainly African. Mycological Papers 117, 8-31.

Dennis RWG. 1970 - Fungus Flora of Venezuela and Adjacent Countries. Kew Bulletin Additional Series III. 1-531.

Dingley JM, Fullerton RA, McKenzie EHC. 1981 - Survey of Agricultural Pests and Diseases. Technical Report Volume 2. Records of Fungi, Bacteria, Algae, and Angiosperms Pathogenic on Plants in Cook Islands, Fiji, Kiribati, Niue, Tonga, Tuvalu, and Western Samoa. South Pacific Bureau for Economic Co-operation. United Nations Development Programme. Food and Agriculture Organization of the United Nations. Rome, 1-485.

Doilom M, Shuttleworth L, Roux J, Chukeatirote E, Hyde KD. 2014 - Barriopsis tectonae sp. nov. a new species of Botryosphaeriaceae from Tectona grandis (teak) in Thailand. Phytotaxa 176, 81-91.

Doilom M, Shuttleworth L, Roux J, Chukeatirote E, Hyde KD. 2015 - Botryosphaeriaceae associated with Tectona grandis (teak) in Northern Thailand. Phytotaxa 233, 1-26.

Doilom M, Dissanayake AJ, Wanasinghe DN, Boonmee S, Liu JK, Bhat DJ, Taylor JE, Bahkali AH, McKenzie EHC, Hyde KD. 2016 - Microfungi on Tectona grandis (teak) in Northern Thailand. Fungal Diversity (online) DOI: 10.1007/s 13225-016-0368-7.

Dugan FM, Schubert K, Braun U. 2004 - Check-list of Cladosporium names. Schlechtendalia 11, $1-103$.

Ebbels DL, Allen DJ. 1979 - A supplementary and annotated list of plant diseases, pathogens and associated fungi in Tanzania. Phytopathological Paper 22, 1-89.

Farr DF, Rossman AY. 2016 - Fungal Databases, Systematic Mycology and Microbiology Laboratory, ARS, USDA. (SMML database - https//nt.ars-grin.gov/fungaldatabases/. Accessed on September 25, 2016).

Firmino AC, Tozze Jr HJ, Furtado EL. 2012 - First report of Ceratocystis fimbriata causing wilt in Tectona grandis in Brazil. New Disease Reports 25, 24. 
Ge Q, Chen Y, Xu T. 2009 - Flora Fungorum Sinicorum. Vol. 38. Pestalotiopsis. Science Press, Beijing, 1-235.

Gezahgne A, Coetzee MPA, Wingfield BD, Wingfield MJ, Roux J. 2004 - Identification of the Armillaria root rot pathogen in Ethiopian plantations. Forest Pathology 34, 133-145.

Giatgong P. 1980 - Host Index of Plant Diseases in Thailand. Second Edition. Mycology Branch, Plant Pathology and Microbiology Division, Department of Agriculture and Cooperatives, Bangkok, Thailand 118.

Goos RD, Gowing DP. 1992 - Type specimens of fungi maintained at herbarium pacificum, Bernice P. Bishop Museum, Honolulu. Mycotaxon 43, 177-198.

Hiratsuka N, Chen ZC. 1991 - A list of Uredinales collected from Taiwan. Transactions of the Mycological Society of Japan 32, 3-22.

Hosagoudar VB. 1988 - Uredinales of Kerala. Journal of Economic and Taxonomic Botany 12, $265-272$.

Hosagoudar VB. 2011 - The genus Schiffnerula in India. Pl. Pathol \& Quarantine 1(2), 131-204.

Index Fungorum 2016 - http,//www.indexfungorum.org/names/names.asp. Accessed on January 2016.

Ito S. 1950 - Mycological Flora of Japan. Vol. II. Basidiomycetes. No. 3. Uredinales Pucciniaceae. Uredinales Imperfecti. Yokendo Ltd., Tokyo, 1-435.

Jimu L, Wingfield MJ, Mwenje E, Roux J. 2015 - Diseases on Eucalyptus species in Zimbabwean plantations and woodlots. Southern Forests 77, 221-230.

Kaneko S, Pham TQ, Hiratsuka Y. 2007 - Notes on some rust fungi in Vietnam. Mycoscience 48, 263-265.

Kamal, 2010 - Cercosporoid Fungi of India. Bishen Singh Mahendra Pal Singh, Dehra Dun, India, $1-352$.

Kranz J. 1965 - Fungi collected in the Republic of Guinea, III. Collections from the Kindia area in 1963/64, and Host Index. Sydowia 19, 92-107.

Lackner M, de Hoog GS. 2011 - Parascedosporium and its relatives: phylogeny and ecological trends. IMA Fungus 2, 39-48.

Li GJ, Hyde KD, Zhao RL, Hongsanan S, Abdel-Aziz FA, Abdel-Wahab MA, Alvarado P, AlvesSilva G, Ammirati JF, Ariyawansa HA, Baghela A, Bahkali AH, Beug M, Bhat DJ, Bojantchev D, Boonpratuang T, Bulgakov TS, Camporesi E, Boro MC, Ceska O, Chakraborty D, Chen JJ, Chethana KWT, Chomnunti P, Consiglio G, Cui BK, Dai DQ, Dai YC, Daranagama DA, Das K, Dayarathne MC, Crop ED, De Oliveira RJV, de Souza CAF, de Souza JI, Dentinger BTM, Dissanayake AJ, Doilom M, Drechsler-Santos ER, GhobadNejhad M, Gilmore SP, Góes-Neto A, Gorczak M, Haitjema CH, Hapuarachchi KK, Hashimoto A, He MQ, Henske JK, Hirayama K, Iribarren MJ, Jayasiri SC, Jayawardena RS, Jeon SJ, Jerônimo GH, Jesus AL, Jones EBG, Kang JC, Karunarathna SC, Kirk PM, Konta S, Kuhnert E, Langer E, Lee HS, Lee HB, Li WJ, Li XH, Liimatainen K, Lima DX, Lin CG, Liu JK, Liu XZ, Liu ZY, Luangsa-ard JJ, Lücking R, Lumbsch HT, Lumyong S, Leaño EM, MaranoAV, Matsumura M, McKenzie EHC, Mongkolsamrit S, Mortimer PE, Nguyen TTT, Niskanen T, Norphanphoun C, O’Malley MA, Parnmen S, Pawłowska J, Perera RH, Phookamsak R, Phukhamsakda C, Pires-Zottarelli CLA, Raspé O, Reck MA, Rocha SCO, de A. Santiago ALCM, Senanayake IC, Setti L, Shang QJ, Singh SK, Sir EB, Solomon KV, Song J, Srikitikulchai P, Stadler M, Suetrong S, Takahashi H, Takahashi T, Tanaka K, Tang LP, Thambugala KM, Thanakitpipattana D, Theodorou MK, Thongbai B, Thummarukcharoen T, Tian Q, Tibpromma S, Verbeken A, Vizzini A, Vlasák J, Voigt K, Wanasinghe DN, Wang Y, Weerakoon G, Wen HA, Wen TC, Wijayawardene NN, Wongkanoun S, Wrzosek M, Xiao YP, Xu J, Yan JY, Yang J, Yang SD, Hu Y, Zhang JF, Zhao J, Zhou LW, Peršoh D, Phillips AJL, Maharachchikumbura SSN. 2016 - Fungal diversity notes 253-366, taxonomic and phylogenetic contributions to fungal taxa. Fungal Diversity 78, 1-237. 
Lorsuwan C, Tontyaporn S, Visarathanonth N, Manoch L, Kakishima M. 1984 - Materials for the rust flora in Thailand I. Transactions of the Mycological Society of Japan 25, 57-65.

Lu B, Hyde KD, Ho WH, Tsui KM, Taylor JE, Wong KM, Yanna, Zhou D. 2000 - Checklist of Hong Kong Fungi. Fungal Diversity Press, 1-207.

Maharachchikumbura SSN, Hyde KD, Jones EBG, McKenzie EHC, Bhat DJ, Dayarathne M, Huang SK, Norphanphoun C, Senanayake IC, Perera RH, Shang Q, Xiao Y, D'souza MJ, Hongsanan S, Jayawardena RS, Daranagama DA, Konta S, Goonasekara ID, Zhuang WY, Jeewon R, Phillips AJL, Abdel-Wahab MA, Al-Sadi AM, Bahkali AH, Boonmee S, Boonyuen N, Cheewangkoon R, Dissanayake AJ, Kang J, Liu JK, Liu X, Liu ZY, Pang KL, Phookamsak R, Promputtha I, Suetrong S, Wen T, Wijayawardene NN. 2016 - Families of Sordariomycetes. Fungal Diversity 79, 1-317.

Mathur RS. 1979 - The Coelomycetes of India. Bishen Singh Mahendra Pal Singh, Delhi, India., 148.

Matsushima T. 1980 - Saprophytic Microfungi from Taiwan, Part 1. Hyphomycetes. Matsushima Mycological Memoirs No. 1, 1-82.

McKenzie EHC, Buchanan PK, Johnston PR. 2005 - Checklist of fungi on cabbage trees (Cordyline spp.) and New Zealand flaxes (Phormium spp.) in New Zealand. New Zealand Journal of Botany 43, 119-139.

Meeboon J, Hidayat I, To-anun C. 2007 - An annotated list of cercosporoid fungi in Northern Thailand. Journal of Agricultural Technology 3, 51-63.

Misra SN, BM, Tiwari RK, Sehgal HS. 1989 - New records of fungi and new host records from India. Indian Forester 115, 40-43.

Mohd Farid A, Lee SS, Mohd Rosli H, Maziah Z, Norwati M. 2005a - Incidence of teak basal root rot caused by Phellinus noxius in Malaysia. Australasian Plant Pathology 34, 277-278.

Mohd Farid A, Lee SS, Maziah Z, Rosli H, Norwati M. 2005b - Basal Root Rot, a new Disease of Teak (Tectona grandis) in Malaysia caused by Phellinus noxius. Malaysian Journal of Microbiology 1, 40-45.

Murali TS, Suryanarayanan TS, Venkatesan G. 2007 - Fungal endophyte communities in two tropical forests of southern India, diversity and host affiliation. Mycological Progress 6, 191-199.

Ono Y, Hennen JF. 1983 - Taxonomy of the Chaconiaceous genera (Uredinales). Transactions of the Mycological Society of Japan 24, 369-402.

Owusu EO. 2011 - Role of intercrops in proliferation of Armillaria rootrot of teak [Tectona grandis (Linn.f.)] in taungya plantation, A case study at the opro forest reserve. BSc Thesis. Kwame Nkrumah University of Science and Technology, Kumasi, Ghana.

Pande A, Rao VG. 1995 - The genus Aplosporella Speg. (= Haplosporella Speg.) (Coelomycetes) from India. Nova Hedwigia 60, 79-117.

Pande A. 2008 - Ascomycetes of Peninsular India. Scientific Publishers (India), Jodhpur, 1-584.

Paul YS, Thakur VK. 2006 - Indian Erysiphaceae. Scientific Publishers (India), Jodhpur, 1-134.

Peregrine WTH, Siddiqi MA. 1972 - A revised and annotated list of plant diseases in Malawi. Phytopathological Papers 16, 1-51.

Pérez M, Lopez MO, Marti O. 2009 - Olivea tectonae, leaf rust of teak, occurs in Cuba. Plant Pathology 58, 1-397.

Piepenbring M. 2006 - Checklist of fungi in Panama. Puente Biológico (Revista Científicade la Universidad Autónoma de Chiriquí) 1, 1-190.

Raabe RD, Conners IL, Martinez AP. 1981 - Checklist of plant diseases in Hawaii. College of Tropical Agriculture and Human Resources, University of Hawaii. Information Text Series No. 22. Hawaii Inst. Tropical Agriculture and Human Resources, 1-313.

Ragunathan AN, Ramakrishnan K. 1972 - Rust fungi of Madras State. I. Mysore Journal of Agricultural Sciences 6, 285-299.

Rajak RC, Pandey AK. 1985 - Fungi from Jabalpur-II. Indian Journal of Mycology and Plant Pathology 15, 186-194. 
Reinking OA. 1920 - Higher basidiomycetes from the Philippines and their hosts, II. Philippine Journal of Science 16, 167-179.

Riley EA. 1960 - A revised list of plant diseases in Tanganyika Territory. Mycological Papers 75, $1-42$.

Rossman AY, Samuels GJ, Lechat C, Chaverri P, Herrera CS. 2013 - Revision of the genus Corallomycetella with Corallonectria gen. nov. for Corallomycetella jatrophae (Nectriaceae, Hypocreales). Mycosystema 32, 518-544.

Sarbhoy AK, Agarwal DK. 1990 - Descriptions of Tropical Plant Pathogenic Fungi. Set 1 to 10. Malhotra Publ. House, New Delhi.

Sawada K. 1959 - Descriptive catalogue of Taiwan (Formosan) fungi. XI. Special Publ Coll Agric Natl Taiwan Univ 8, 1-268.

Shaw DE. 1984 - Microorganisms in Papua New Guinea. Research Bulletin, Department of Primary Industry, Papua New Guinea 33, 123.

Shivas RG, Athipunyakom P, Likhitekaraj S, Butranu W, Bhasabutra T, Somrith A, Vánky K \&Vánky C. 2007 - An annotated checklist of smut fungi (Ustilaginomycetes) from Thailand. Australasian Plant Pathology 36, 376-382.

Sivanesan A. 1975 - Redisposition and descriptions of some Amphisphaeria species and a note on Macrovalsaria. Transactions of the British Mycological Society 65, 395-402.

Spaulding P. 1961 - Foreign Diseases of Forest Trees of the World. U.S.D.A. Agriculture Handbook 197, 1-361.

Stevens FL. 1925 - Hawaiian Fungi. Bernice P. Bishop Mus Bull 19, 1-189.

Stevenson JA. 1975 - Fungi of Puerto Rico and the American Virgin Islands. Contribution of Reed Herbarium 23, 1-743.

Tai FL. 1979 - Sylloge Fungorum Sinicorum. Science Press, Academica Sinica, Peking, 1-1527.

Tianyu Z. 2009 - Flora Fungorum Sincorum Vol. 31, 26 Genera of Dematiaceous Dictyosporous Hyphomycetes Excluding Alternaria. Science Press, Beijing 31, 1-231.

Teodoro NG. 1937 - An Enumeration of Philippine Fungi. Techn. Bull. Dept. Agric. Comm. Manila, Technical Bulletin 4, 1-585.

Thaung MM. 2007a - Powdery mildews in Burma with reference to their global host-fungus distributions and taxonomic comparisons. Australasian Plant Pathology 36, 543-551.

Thaung MM. 2007b - A preliminary survey of macromycetes in Burma. Australasian Mycologist $26,16-36$.

Thaung MM. 2008 - Biodiversity survey of coelomycetes in Burma. Australasian Mycologist 27, 74-110.

Thirumalachar MJ. 1949 - Telia of the leaf-rust on teak. Current Science 18, 175-177.

To-anun C, Hidayat I, Meeboon J. 2011 - Genus Cercospora in Thailand, Taxonomy and Phylogeny (with a dichotomous key to species). Plant Pathology \& Quarantine 1, 11-87.

Udayanga D, Liu X, McKenzie EHC, Chukeatirote E, Hyde KD. 2012a - Multi-locus phylogeny reveals three new species of Diaporthe from Thailand. Cryptogamie Mycologie 33, 295309.

Udayanga D, Liu X, Crous PW, McKenzie EHC, Chukeatirote E, Hyde KD. 2012b - A multi-locus phylogenetic evaluation of Diaporthe (Phomopsis). Fungal Diversity 56, 157-171.

Urtiaga R. 1986 - Indice de enfermedades en plantas de Venezuela y Cuba. Impresos en Impresos Nuevo Siglo. S.R.L., Barquisimeto, Venezuela, 1-201.

Urtiaga R. 2004 - Indice de enfermedades en plantas de Venezuela y Cuba, Second Edition. Unknown journal or publisher, 1-301.

Vasudeva RS. 1963 - Indian Cercosporae. Indian Council of Agricultural Research, New Delhi, 1245.

Vrijmoed LLP, Hodgkiss IJ, Thrower LB. 1982 - Factors affecting the distribution of lignicolous marine fungi in Hong Kong. Hydrobiologia 87, 143-160. http//dx.doi.org/10.1007 /BF00015195.

Vrijmoed LLP, Hodgkiss IJ, Thrower LB. 1986 - Occurrence of fungi on submerged pine and teak 
blocks in Hong Kong coastal waters. Hydrobiologia 135, 109-122. http//dx.doi. org/10.1007/BF00006463.

West J. 1938 - A preliminary list of plant diseases in Nigeria. Kew Bull. 1, 17-23.

Whitton SR, McKenzie EHC, Hyde KD. 2012 - Fungi Associated with Pandanaceae. Fungal Diversity Research Series 21, 1-429.

Wiehe PO. 1948 - The plant diseases and fungi recorded from Mauritius. Mycological Papers 24, 1-39.

Wikee S, Lombard L, Crous PW, Nakashima C, Motohashi K, Chukeatirote E, Alias SA, McKenzie EHC, Hyde KD. 2013a - Phyllosticta capitalensis, a widespread endophyte of plants. Fungal Diversity 60, 91-105.

Wikee S, Lombard L, Nakashima C, Motohashi K, Chukeatirote E, Cheewangkoon R, McKenzie EHC, Hyde KD, Crous PW. 2013b - A phylogenetic re-evaluation of Phyllosticta (Botryosphaeriales). Studies in Mycology 76, 1-29.

Yen JM, Kar AK, Das BK. 1982 - Studies on hyphomycetes from West Bengal, India, II. Cercospora and allied genera of West Bengal, 2. Mycotaxon 16, 58-79.

Zhao GZ, Zhang TY. 2007 - Species of the genus Monodictys (Hyphomycetes) from China. Mycosystema 26, 324-335.

Zhuang JY, Wei SX. 1999 - Fungal flora of tropical Guangxi, China, a preliminary checklist of rust fungi. Mycotaxon 72, 377-388.

Zhuang WY. 2001 - Higher Fungi of Tropical China. Mycotaxon, Ltd., Ithaca, NY, 1-485. 